\title{
Dipeptidyl Peptidase-4 Involved in Regulating Mitochondria Function in Cardiomyocytes through Nrf2 and PGC-1a Signaling
}

Shih-Yi Lee

Mackay Memorial Hospital

Shao-Tung Wu

Fu Jen Catholic University

Ming-Jai Su

National Taiwan University

Yao-Jen Liang

Fu Jen Catholic University

Hui-Chun Ku ( $\nabla$ jessiku@gmail.com )

Fu Jen Catholic University

Original investigation

Keywords: DPP4, oxidative stress, mitochondria, Nrf2, PGC-1a

Posted Date: June 4th, 2020

DOI: https://doi.org/10.21203/rs.3.rs-30722/v1

License: (c) (1) This work is licensed under a Creative Commons Attribution 4.0 International License. Read Full License 


\section{Abstract}

Background: Oxidative stress is an imbalance between the production of reactive oxygen species (ROS) and the detoxification ability of reactive intermediates. It will lead to mitochondrial damage and dysfunction, resulting in the dysfunction of bioenergetic control and loss of ATP production, which is contributed to the pathogenesis of cardiac diseases. Dipeptidyl peptidase-4 (DPP4) is a transmembrane glycoprotein ubiquitously expressed and has multifunctional properties. DPP4 inhibitors are a class of oral diabetes drugs that inhibit the enzyme activity. In addition to its enzymatic property, considerably less is known regarding the nonenzymatic function of DPP4.

Methods: We knocked down DPP4 gene expression in cultured cardiomyocytes to exclude any external and enzymatic substrate effects and compared the response between DPP4 knockdown and wild-type cardiomyocytes in response to oxidative stress.

Results: $\mathrm{H}_{2} \mathrm{O}_{2}$-induced oxidative stress-stimulated intracellular and mitochondrial ROS concentration led to the loss of mitochondrial function, ATP production, and increased Bax and cleaved PARP expression, resulting in the loss of cell viability in cardiomyocytes. Oxidative stress induced DPP4 expression. Knocking down DPP4 ameliorated $\mathrm{H}_{2} \mathrm{O}_{2}$-induced loss of cell viability by preserving mitochondrial bioenergy, reducing intracellular ROS production, alleviating apoptosis-associated protein expression. Knocking down DPP4 increased its capability against oxidative stress by enhancing Nrf2 and PGC-1a signaling, which is associated with preserving mitochondrial function.

Conclusions: DPP4 is a mediator of oxidative stress. Knocking down DPP4 without any external substrate mediators increased the capability of cardiomyocytes against oxidative stress, which indicated that DPP4 mediated more than the enzymatic-dependent pathway.

\section{Background}

Oxidative stress is an imbalance between the production of reactive oxygen species (ROS) and the detoxification ability of reactive intermediates [1]. Excessive ROS production, which is caused by several factors such as hypoxia, cell ischemia, or toxin exposure, leads to harmful effects including DNA damage, lipid peroxidation, protein denaturation and dysfunction, and cell death [1, 2]. A constant supply of oxygen is indispensable for cardiac viability and function [3]. Oxygen is a major determinant of cardiac gene expression and a critical participant in ROS formation [3]. Therefore, ROS production is highly regulated in the heart. Dysregulation of ROS production has been implicated in various cardiac diseases [4], including diabetic cardiomyopathy, cardiac hypertrophy, and ischemia-reperfusion injury [5,6]. These may lead to heart failure [6]. Understanding the signal transduction mechanism underlying ROS during cardiac dysfunction is crucial.

The heart has massive energy requirements [7]. Mitochondria generate a large amount of adenosine triphosphate (ATP) to maintain cellular and critical functions for regulating the energy metabolism [7], and controlling intracellular signaling, apoptosis, and the redox state [8]. Mitochondria are the main 
organelles responsible for ROS production [9], which is a byproduct of the respiratory chain during oxidative phosphorylation [8]. Superoxide radicals are the primary species produced by mitochondria and may be produced by the electron transport chain, including complexes I and III [10,11]. Superoxide dismutases (SOD) facilitate $\mathrm{H}_{2} \mathrm{O}_{2}$ formation from superoxide radicals [12]. $\mathrm{H}_{2} \mathrm{O}_{2}$ is another major chemical mediator of ROS. Catalase produces water and oxygen from $\mathrm{H}_{2} \mathrm{O}_{2}$ [13]. Under pathophysiological conditions, the excessive production of superoxide radical will induce the overloading of both ROS scavenger and antioxidant systems [3]. This will lead to mitochondrial damage and dysfunction, resulting in the dysfunction of bioenergetic control and loss of ATP production, which is contributed to the pathogenesis of cardiac diseases [14].

Dipeptidyl peptidase-4 (DPP4) is a transmembrane glycoprotein expressed in various cell types and has multifunctional properties [15]. DPP4 has peptidase activity that involves cleaving $\mathrm{N}$ terminal dipeptides, which contain proline or alanine at the second position of peptides [16]. DPP4 substrates include glucagon-like peptide-1 (GLP-1), growth factors, chemokines, neuropeptides, and vasoactive peptides [17]. Several DPP4 inhibitors and the GLP-1 analog are being developed as a class of antihyperglycemic agents to treat diabetes [16]. Pharmacological inhibition or genetic deficiency of DPP4 leads to prolonged GLP-1 activity [18-20], which prevented the activation of cell death signaling in cardiomyocytes and improved cardiac function in rodents with cardiac diseases. [19, 21, 22]. In addition to its enzymatic property, considerably less is known regarding the nonenzymatic function of DPP4. DPP4 is expressed in the heart and has a complicated role [20]. Our previous study revealed that glucose uptake increased in cardiomyocytes isolated from DPP4-deficient rats that had a single mutation on the DPP4 enzymatic site, although this phenomenon was not found in cardiomyocytes isolated from wild-type rats treated with a short-term DPP4 inhibitor [23]. Cardiomyocytes in DPP4-deficient rats may already interact with various growth factors, cytokines, and neurohumoral factors before isolation, which may mask the primary action of DPP4. Therefore, in this study, we knocked down DPP4 gene expression in cultured cardiomyocytes to exclude any external and enzymatic substrate effects and compared the response between DPP4 knockdown and wild-type cardiomyocytes in response to oxidative stress. Furthermore, investigating the role of mitochondria is currently an area of great interest. Whether DPP4 plays a role in regulating mitochondrial function was investigated in this study.

\section{Materials And Methods}

\section{Cell culture}

$\mathrm{HL}-1$, a mouse atrial cardiomyocyte, is used in this study and obtained from Dr. William C. Claycomb (Louisiana State University Health Sciences Center, New Orleans, LA). HL-1 cells can be serially passaged, maintain the ability to contract and retain differentiated cardiac morphological, biochemical, and electrophysiological properties. The cells were cultured in Claycomb medium supplemented with $10 \%$ FBS (Gibco, Scotland, UK), 2 mM L-glutamine (Gibco, Scotland, UK), 0.1 mM norepinephrine and 
antibiotics $\left(100 \mu \mathrm{g} / \mathrm{ml}\right.$ penicillin and $100 \mu \mathrm{g} / \mathrm{ml}$ streptomycin) at $37^{\circ} \mathrm{C}$ under a $5 \%$ CO2 air atmosphere. $\mathrm{H}_{2} \mathrm{O}_{2}(200 \mu \mathrm{M})$ was added to induce oxidative stress for $24 \mathrm{~h}$.

\section{Lentiviral transduction for gene knockdown}

Lentiviral particles containing shRNAs pLK0.1 (\#TRCN0000031289) was used to knockdown DPP4 in HL1 cardiomyocytes. Lentivirus containing scrambled shRNA, pLKO-shScr (\#TRCN00001) was used as nontargeting control and served as wild-type. HL-1 cardiomyocytes were transduced with lentiviral vectors with $\mathrm{MOI}$ of 15, along with $8 \mathrm{ug} / \mathrm{mL}$ Polybrene (hexadimethrine bromide, Sigma Aldrich, MO, USA) in medium supplemented with $1 \%$ FBS for $24 \mathrm{hr}$ and then replaced with fresh medium. Transduced HL-1 cardiomyocytes were treated with culture media containing puromycin at final concentration of $5 \mathrm{ng} / \mathrm{ml}$, for selection of transduced cells.

\section{RNA extraction and reverse transcription quantitative polymerase chain reaction (RT-qPCR)}

Total RNA was isolated from cells using TRIzol (Thermo Fisher Scientific, MA, USA). Total RNA was reverse transcribed with Maxima First Strand cDNA Synthesis Kit (Thermo Fisher Scientific, MA, USA) and SYBR Green was used for performing quantitative real time PCR. The expression level of each individual transcript was normalized to HPRT gene and expressed relative to the mean expression values of wildtype samples.

\section{Detection of cell viability}

Cell viability was determined by a 3-(4,5-Dimethylthiazol-2-yl)-2,5-diphenyltetrazoliumbromide (MTT) assay. Cells were treated with MTT at $0.5 \mathrm{mg} / \mathrm{ml}$. The purple formazan crystals were dissolved in DMSO. Solutions were then loaded into a 96 well plate, and the absorbance was determined on an automated microplate spectrophotometer at $570 \mathrm{~nm}$.

\section{Detection of ROS production}

Intracellular ROS and mitochondria superoxide generation were detected in HL-1 cardiomyocytes by labeling with fluorescence dye dihydroethidium (DHE) and MitoSOX (Thermo Fisher Scientific, MA, USA), respectively. Cells were loaded with $10 \mu \mathrm{M}$ dye at $37^{\circ} \mathrm{C}$ for $30 \mathrm{~min}$, washed with PBS. By using fluorescence microscopy, DHE and MitoSOX were monitored at $510 \mathrm{~nm}$ excitation and $580 \mathrm{~nm}$ emission, respectively. Fluorescence intensity was calculated by averaging fluorescence intensity of numerous outlined cells using ImageQuant (Molecular Dynamics, Inc., Sunnyvale, CA, USA).

\section{Detection of mitochondrial membrane potential}

Mitochondrial membrane potential was detected in $\mathrm{HL}-1$ cardiomyocytes by labeling with fluorescence dye tetramethylrhodamine (TMRM) (Thermo Fisher Scientific, MA, USA). Cells were loaded with $10 \mu \mathrm{M}$ dye at $37^{\circ} \mathrm{C}$ for $30 \mathrm{~min}$, washed with PBS. By using fluorescence microscopy, TMRM was monitored at $548 \mathrm{~nm}$ excitation and $574 \mathrm{~nm}$ emission, respectively. Fluorescence intensity was calculated by 
averaging fluorescence intensity of numerous outlined cells using ImageQuant (Molecular Dynamics, Inc., Sunnyvale, CA, USA).

\section{Detection of mitochondrial bioenergetic function}

A Seahorse metabolic flux analyzer was used for measuring the rate of oxidative phosphorylation, according to methods previously described [24]. For measuring oxygen consumption rate (OCR), Seahorse XF Cell Mito Stress Test kit (Agilent Technologies, Inc., Santa Clara, CA, USA) including different pharmacological inhibitors were used to probe the function of individual components in the respiratory chain. A seeding density of 30,000 cells/well was used to detect the changes in OCR due to exposure with $\mathrm{H}_{2} \mathrm{O}_{2}$. The cells were incubated in XF24 culture microplates with culture medium for $24 \mathrm{~h}$ as well as the sensor cartridge hydrated in XF Calibrant at $37^{\circ} \mathrm{C}$ in a non-CO2 incubator overnight. Prior to all bioenergetic assays, the culture medium was replaced with unbuffered medium. To estimate the basal oxygen consumption rate (OCR) coupled to ATP synthesis, 1uM oligomycin was injected to inhibit the ATP synthase. The decreased OCR in response to oligomycin indicated the cells were using mitochondria to generate ATP. To determine the maximal OCR that the cells could sustain, 0.5 uM carbonyl cyanide-4(trifluoromethoxy) phenylhydrazone (FCCP) was injected, which made the mitochondrial inner membrane permeable to protons. The deduction between maximal OCR and the basal OCR is the spare capacity of mitochondria. Finally, 0.5uM antimycin A and rotenone was injected to inhibit electron flux. The remaining OCR could be ascribed to oxygen consumption due to the formation of mitochondrial ROS and non-mitochondrial sources.

\section{Protein extraction}

A nucleus isolation kit (Abcam,USA) and the multiple centrifugation method were used to perform protein extraction of nuclear fractions, according to methods previously described [25]. The total fraction or the nucleus fraction of protein were isolated by an extraction buffer (Thermo Fisher Scientific Inc., IL, USA) containing a cocktail protease and phosphatase inhibitor (Sigma, St. Louis, MO, USA). A BCA protein assay kit (Thermo Fisher Scientific Inc.) was used to determine the protein concentration.

\section{Determination of protein expression}

A Western blotting technique was performed to detect protein expression. Equal quantities of proteins were first denatured for $10 \mathrm{~min}$ in boiling sample buffer $(31.3 \mathrm{mM}$ Tris- $\mathrm{HCl}$ at $\mathrm{pH} 6.8,25 \%$ glycerol, 10\% sodium dodecyl sulfate (SDS), 10\% 2-mercaptoethanol, and $0.00125 \%$ bromophenol blue). Then, proteins were separated using SDS-polyacrylamide gel electrophoresis and transferred to polyvinylidene difluoride membranes (Perkin-Elmer Life Sciences, Boston, MA, USA). The membranes were blocked with $5 \%$ fat-free milk dissolved in Tris-buffered saline with Tween 20 (TBST) and incubated overnight with the primary antibodies of bax, cleaved Poly (ADP-ribose) polymerase (PARP), nuclear factor erythroid 2related factor 2 (Nrf2), peroxisome proliferator-activated receptor gamma coactivator 1-alpha (PGC-1区), lamin (Abcam, USA), and glyceraldehyde 3-phosphate dehydrogenase (GAPDH) (Santa Cruz Biotechnology, CA, USA) at $4{ }^{\circ} \mathrm{C}$. Subsequently, the membranes were washed several times with TBST, which was followed by incubation with horseradish-peroxidase-conjugated secondary antibodies (Santa 
Cruz Biotechnology, Inc.) for 1 hour. After washing several times, the protein signals were detected using an enhanced chemiluminescence system (Millipore, Bedford, MA, USA). The blots were scanned and quantified using Imagequant (Molecular Dynamics, Inc., Sunnyvale, CA, USA).

\section{Statistical analyses}

All values are represented as mean \pm standard error. The results were analyzed using one-way ANOVA, followed by Bonferroni post hoc tests. We considered that $p<0.05$ to be significant.

\section{Results}

\section{Knocking down DPP4 expression attenuated $\mathrm{H}_{2} \mathrm{O}_{2}$-induced loss of cell viability in cardiomyocytes}

To confirm DPP4 expression in knocked down cardiomyocytes, the mRNA expression of DPP4 in cardiomyocytes was measured. DPP4 expression in mRNA was determined using RT-qPCR (Fig. 1A). DPP4 mRNA expression was considerably lower in knocked down cardiomyocytes than in wild-type cardiomyocytes. After exposure to $200 \mathrm{uM}$ of $\mathrm{H}_{2} \mathrm{O}_{2}$ for $24 \mathrm{~h}$, the mRNA expression of DPP4 was upregulated in wild-type cardiomyocytes but was not detected in DPP4-knocked down cardiomyocytes. To investigate whether DPP4 influenced $\mathrm{H}_{2} \mathrm{O}_{2}$-induced oxidative stress in cardiomyocytes, a MTT assay was used to measure cell viability. Exposure to $\mathrm{H}_{2} \mathrm{O}_{2}$ exhibited an inhibitory response on cell viability while knocking down DPP4-recovered cell viability (Fig. 1B). These results suggested that DPP4 affects the regulation of oxidative stress in cardiomyocytes.

\section{Knocking down DPP4 alleviated intracellular ROS production under oxidative stress}

To determine whether DPP4 affects intracellular ROS production under oxidative stress, cardiomyocytes were labeled using DHE (red). DHE staining indicated that the intracellular ROS concentration was induced by $\mathrm{H}_{2} \mathrm{O}_{2}$; however, knocking down DPP4 alleviated the intracellular ROS concentration in cardiomyoyctes (Fig. 2).

To investigate the effect of DPP4 on mitochondrial ROS production during oxidative stress, cardiomyocytes were labeled with mitoSOX (red). MitoSOX staining indicated that the mitochondrial ROS concentration was induced by $\mathrm{H}_{2} \mathrm{O}_{2}$; however, knocking down DPP4 alleviated the mitochondrial ROS concentration in cardiomyoyctes (Fig. 3).

\section{Knocking down DPP4 preserved mitochondrial membrane potential under oxidative stress}


To determine whether DPP4 affects mitochondrial membrane potential, cardiomyocytes were labeled with TMRM (red). TMRM staining indicated that mitochondrial membrane potential was reduced by $\mathrm{H}_{2} \mathrm{O}_{2}$; however, knocking down DPP4 preserved mitochondrial membrane potential in cardiomyoyctes (Fig. 4).

\section{Knocking down DPP4 recovered mitochondrial bioenergetics function under oxidative stress}

To assess cellular bioenergetics in intact cardiomyocytes, the Seahorse metabolic flux analyzer was used to determine oxygen consumption rates (Fig. 5). Cardiomyocytes were treated with $\mathrm{H}_{2} \mathrm{O}_{2}$ before the measurement of OCR. No significant differences in the basal respiratory rate of wild-type and DPP4 knocked down cardiomyocytes were observed. $\mathrm{H}_{2} \mathrm{O}_{2}$ reduced the basal OCR in cardiomyocytes (Fig. 5A and $B$ ), which suggested that $\mathrm{H}_{2} \mathrm{O}_{2}$ inhibits mitochondrial aerobic respiration. Knocking down DPP4 preserved the basal respiratory rate under oxidative stress. Afterward, cardiomyocytes were treated with $1 \mu \mathrm{M}$ oligomycin, $0.5 \mu \mathrm{M} \mathrm{FCCP}$, and $0.5 \mu \mathrm{M}$ rotenone plus antimycin $\mathrm{A}$ to determine the different parameters of mitochondrial bioenergetics function. $\mathrm{H}_{2} \mathrm{O}_{2}$ reduced ATP-link respiratory production (Fig. 5A and C), maximal respiration capacity (Fig. 6A and D), and spare respiration capacity (Fig. 5A and $\mathrm{E}$ ) in the wild-type cardiomyocytes under oxidative stress. By contrast, knocking down DPP4 significantly reversed the dysfunction of aerobic metabolism in mitochondria induced by $\mathrm{H}_{2} \mathrm{O}_{2}$.

\section{Knocking down DPP4 affected the expression of oxidative stress-associated protein}

To investigate the effect of DPP4 on the cell death-associated protein, Western blotting was performed to detect several proteins levels. Several apoptotic-associated proteins were detected. Bax is a proapoptotic protein [26]. Cleavage of PARP-1 from caspases indicates apoptosis [27], while the primary function of PARP-1 is to detect and repair DNA damage [28]. $\mathrm{H}_{2} \mathrm{O}_{2}$ induced bax and cleaved PARP protein expression in wild-type cardiomyocytes (Fig. 6A, B, and C). The elevation of apoptotic-associated proteins was alleviated by knocking down DPP4 under oxidative stress.

To determine the effect of DPP4 on antioxidant and mitochondrial bioenergy-associated proteins, the expression of Nrf2 and PGC-1】 was measured. Nrf2 is a key transcription factor that regulates antioxidant defense [29], and PGC-1区 is a transcriptional coactivator that is a central inducer of mitochondrial biogenesis in cells [30]. The Nrf2 level was induced after $\mathrm{H}_{2} \mathrm{O}_{2}$ exposure (Fig. 6D and E). Knocking down DPP4 induced Nrf2 protein expression more than wild-type cardiomyocytes did in response to $\mathrm{H} 2 \mathrm{O} 2$. PGC-1区 was upregulated in the basal condition of DPP4-knocked down cardiomyocytes (Fig. 6D and F). The PGC-1》 level was decreased by $\mathrm{H}_{2} \mathrm{O}_{2}$ exposure. Knocking down DPP4 rescued the inhibition of PGC-1『 protein expression induced by $\mathrm{H}_{2} \mathrm{O}_{2}$.

\section{Discussion}


The results of the present study demonstrated that $\mathrm{H}_{2} \mathrm{O}_{2}$-induced oxidative stress-stimulated intracellular and mitochondrial ROS concentration led to the loss of mitochondrial function, ATP production, and increased Bax and cleaved PARP expression, resulting in the loss of cell viability in cardiomyocytes. Oxidative stress induced DPP4 expression. Knocking down DPP4 ameliorated $\mathrm{H}_{2} \mathrm{O}_{2}$-induced loss of cell viability by preserving mitochondrial bioenergy and reducing intracellular ROS production, which suggested the importance of DPP4 for regulating mitochondrial function under oxidative stress. Furthermore, DPP4 knocking down cardiomyocytes enhanced Nrf2 and PGC1a expression after $\mathrm{H}_{2} \mathrm{O}_{2}$ exposure. Knocking down DPP4 without any external substrate mediators increased the capability of cardiomyocytes against oxidative stress, which indicated that DPP4 mediated more than the enzymaticdependent pathway.

The effect of DPP4 inhibitors on the cardiovascular system is controversial [31, 32]. Some meta-analyses have indicated that inhibiting DPP4 activity by using gliptins may reduce the risk of heart failure and other adverse cardiovascular events in patients with diabetes [33]. However, DPP4 inhibition by using saxagliptin has been associated with increased risk of hospitalization for heart failure in patients with diabetes [34]. Conflicting results have also been reported in animal studies. Young euglycemic mice with knocked down DPP4 exhibited a cardioprotective response after pressure overload or doxorubicin administration induced heart failure [35]. By contrast, diabetic mice treated with DPP4 inhibitors exhibited impaired cardiac function and dysregulated inflammatory proteins [35]. However, diabetic mice treated with liraglutide, a clinically available GLP-1 receptor agonist, exhibited preservation of cardiac function [35]. These conflicting results may reflect differential alteration in the levels of substrates and metabolites of DPP4, such as growth factors, cytokines, and neurohumoral factors, which may vary depending on study conditions and participants. This study determined the role of DPP4 beyond external mediator in cardiomyocytes. DPP4 was upregulated and served as a mediator under oxidative stress. The upregulation of DPP4 contributes to the progression of several types of diseases. DPP4 was upregulated in the adipose tissue of obese insulin-resistant participants, which were correlated with insulin resistance and inflammation measures [36]. DPP4 was stimulated by advanced glycation end-product exposure and involved in the inflammatory response of proximal tubular cells [37]. DPP4 was increased in fibroblasts isolated from patients with systemic sclerosis and were involved in the differentiation of fibroblasts into myofibroblasts [38]. Such evidence supports the speculation that DPP4 participates in the regulation of cellular functions and the progression of disease pathologies [39].

Mitochondria are targets and sources of ROS [9]. Oxidative stress promotes mitochondrial dysfunction. The mitochondrial respiratory chain can be impaired by mitochondrial dysfunction, which can lead to excess ROS and exacerbate oxidative stress to form a vicious circle [40]. Increased ROS levels can open the inner membrane anion channel and result in mitochondrial membrane depolarization, which can cause mitochondrial dysfunction and diminish ATP production [11]. Mitochondrial dysfunction initiates cell apoptosis [41], which is regulated by proapoptotic proteins such as Bax and Bak [26]. The proapoptotic proteins are inserted into mitochondrial membranes, where they stimulate apoptotic signal transduction and activate caspase [42]. Caspase mediates apoptosis by cleaving several key proteins 
required for cellular function [42], such as PARP [27]. DPP4 inhibitors induce protective effects on mitochondria. Treatment with vildagliptin or sitagliptin in diabetic rats significantly attenuated brain mitochondrial ROS production and depolarization through the GLP-1 pathway [43]. DPP4 inhibitors improved exercise capacity and mitochondrial biogenesis in mice with heart failure by activating GLP-1 signaling [44]. In our study, beyond GLP-1, knocking down DPP4 alleviated intracellular ROS production and maintained mitochondria membrane potential after $\mathrm{H}_{2} \mathrm{O}_{2}$ exposure, which resulted in the recovery of basal respiration capacity and ATP-linked oxygen consumption. $\mathrm{H}_{2} \mathrm{O}_{2}$-induced loss of mitochondrial maximal and respiration spare capacity were recovered by knocking down DPP4. Decreased Bax expression and cleaved PARP were involved in inhibiting apoptosis in cardiomyocytes with knocked down DPP4. This study indicated that without the participation of GLP-1, loss of DPP4 can preserve mitochondrial bioenergy under oxidative stress.

The regulation of oxidative stress and mitochondrial metabolism is controlled by numerous transcriptional networks. PGC-1》 interacts with transcription factors to exert biological functions [30], and is a positive regulator of mitochondrial biogenesis, respiration, and many other metabolic processes [30]. The expression of PGC-1区 expression is regulated by DPP4. Inhibition of DPP4 by pharmacological compounds was shown to increase PGC-1】 expression $[45,46]$. Knocking down DPP4 also induced the upregulation of PGC-1区 in primary human adipocytes and contributes to adipocyte maturation [47]. Similar results were observed in our study. Knocking down DPP4 stimulated PGC-1区 expression, which may have contributed to the preservation of mitochondrial bioenergy under oxidative stress. In addition, Nrf2 is another transcription factor that regulates the expression of genes involved in regulating redox homeostasis [48]. Free Nrf2 is translocated to the nucleus and binds to antioxidant responsive elements (AREs) of target gene promoters [29]. These genes are codes for cytoprotective properties, including for antioxidant enzymes and anti-inflammatory enzymes [48]. Knocking down DPP4 was shown to further induce the upregulation of Nrf2 expression in response to oxidative stress. These results coincided with those of a previous study in which the protective effect of sitagliptin on acute pancreatitis-associated intestinal inflammation was reported to be abolished in mice with Nrf2 knocked out [49]. Nrf2 is also key for supporting the structural and functional integrity of mitochondria [50], and participates in regulating mitochondrial biogenesis by maintaining PGC-1区 levels with AREs in the gene promoter [51, 52]. A regulatory loop involves PGC-1 $a$ and Nrf2. PGC-1 a controls antioxidant genes through Nrf2 activation [51]. Downregulation of PGC-1 a expression almost completely inhibits Nrf2 binding to the ARE and reduces SOD2 content [51]. PGC-1 a knockout diminishes Nrf2-dependent mitochondrial biogenesis [53]. We discovered that Nrf2 and PGC-1a together preserve bioenergetic capability and maintain cellular function in response to oxidative stress in DPP4-knocked down cardiomyoctyes.

\section{Concussion}

DPP4 is a mediator of oxidative stress. Knocking down DPP4 increased its capability against oxidative stress by enhancing Nrf2 and PGC-1a signaling, which is associated with preserving mitochondrial function. 


\section{List Of Abbreviations}

ROS: Reactive oxygen species

DPP4: Dipeptidyl peptidase-4

ATP: Adenosine triphosphate

SOD: Superoxide dismutases

GLP-1: glucagon-like peptide-1

MTT: 3-(4,5-Dimethylthiazol-2-yl)-2,5-diphenyltetrazoliumbromide

DHE: Dihydroethidium

TMRM: Tetramethylrhodamine

OCR: Oxygen consumption rate

FCCP: Cyanide-4-(trifluoromethoxy) phenylhydrazone

SDS: Sodium dodecyl sulfate

TBST: Tris-buffered saline with Tween 20

PARP: Poly (ADP-ribose) polymerase

Nrf2: Nuclear factor erythroid 2-related factor 2

PGC-1囚: Peroxisome proliferator-activated receptor gamma coactivator 1-alpha

GAPDH: Glyceraldehyde 3-phosphate dehydrogenase

ARE: Antioxidant responsive elements

\section{Declarations}

\section{Ethics approval and consent to participate}

The experimental protocol was reviewed and approved by the local government.

\section{Consent for publication}

All authors gave their consent for publication. 


\section{Availability of data and materials}

All relevant data are within the paper.

\section{Competing interests}

The authors declare that they have no competing interests.

\section{Funding}

We like to thank the research funding from Ministry of Science and Technology, Taiwan (MOST 106-2321B-030-002-MY3), and Mackay Memorial Hospital, Taiwan (MMH-TT-10907).

\section{Authors' contributions}

Conceived and designed the experiments: HCK, SYL, MJS, YJL. Performed the experiments: HCK, SYL, STW. Analyzed the data: HCK, SYL, STW. Contributed reagents/materials/analysis tools: HCK, SYL, MJS, YJL. Wrote the manuscript: HCK, SYL.

\section{Acknowledgements}

We thank for the lentiviral particles from Academia Sinica, Taiwan.

\section{References}

1. Hensley K, Robinson KA, Gabbita SP, Salsman S, Floyd RA. Reactive oxygen species, cell signaling, and cell injury. Free Radic Biol Med. 2000;28(10):1456-62.

2. Pizzino G, Irrera N, Cucinotta M, Pallio G, Mannino F, Arcoraci V, et al. Oxidative Stress: Harms and Benefits for Human Health. Oxid Med Cell Longev. 2017;2017:8416763.

3. Giordano FJ. Oxygen, oxidative stress, hypoxia, and heart failure. J Clin Invest. 2005;115(3):500-8.

4. Peoples JN, Saraf A, Ghazal N, Pham TT, Kwong JQ. Mitochondrial dysfunction and oxidative stress in heart disease. Exp Mol Med. 2019;51(12):1-13.

5. Burgoyne JR, Mongue-Din H, Eaton P, Shah AM. Redox signaling in cardiac physiology and pathology. Circ Res. 2012;111(8):1091-106.

6. Sawyer DB, Siwik DA, Xiao L, Pimentel DR, Singh K, Colucci WS. Role of oxidative stress in myocardial hypertrophy and failure. J Mol Cell Cardiol. 2002;34(4):379-88.

7. Jafri MS, Dudycha SJ, O'Rourke B. Cardiac energy metabolism: models of cellular respiration. Annu Rev Biomed Eng. 2001;3:57-81. 
8. Handy DE, Loscalzo J. Redox regulation of mitochondrial function. Antioxid Redox Signal. 2012;16(11):1323-67.

9. Zorov DB, Juhaszova M, Sollott SJ. Mitochondrial reactive oxygen species (ROS) and ROS-induced ROS release. Physiol Rev. 2014;94(3):909-50.

10. de Grey AD. Reactive oxygen species production in the mitochondrial matrix: implications for the mechanism of mitochondrial mutation accumulation. Rejuvenation Res. 2005;8(1):13-7.

11. Gao L, Laude K, Cai H. Mitochondrial pathophysiology, reactive oxygen species, and cardiovascular diseases. Vet Clin North Am Small Anim Pract. 2008;38(1):137-55, vi.

12. Buettner GR. Superoxide dismutase in redox biology: the roles of superoxide and hydrogen peroxide. Anticancer Agents Med Chem. 2011;11(4):341-6.

13. Heck DE, Shakarjian M, Kim HD, Laskin JD, Vetrano AM. Mechanisms of oxidant generation by catalase. Ann N Y Acad Sci. 2010;1203:120-5.

14. Zhou B, Tian R. Mitochondrial dysfunction in pathophysiology of heart failure. J Clin Invest. 2018;128(9):3716-26.

15. Mentlein R. Dipeptidyl-peptidase IV (CD26)--role in the inactivation of regulatory peptides. Regul Pept. 1999;85(1):9-24.

16. Mulvihill EE, Drucker DJ. Pharmacology, physiology, and mechanisms of action of dipeptidyl peptidase-4 inhibitors. Endocr Rev. 2014;35(6):992-1019.

17. Kim NH, Yu T, Lee DH. The nonglycemic actions of dipeptidyl peptidase-4 inhibitors. Biomed Res Int. 2014;2014:368703.

18. Hausenloy DJ, Whittington HJ, Wynne AM, Begum SS, Theodorou L, Riksen N, et al. Dipeptidyl peptidase-4 inhibitors and GLP-1 reduce myocardial infarct size in a glucose-dependent manner. Cardiovasc Diabetol. 2013;12:154.

19. Ku HC, Chen WP, Su MJ. DPP4 deficiency preserves cardiac function via GLP-1 signaling in rats subjected to myocardial ischemia/reperfusion. Naunyn Schmiedebergs Arch Pharmacol. 2011;384(2):197-207.

20. Sauve M, Ban K, Momen MA, Zhou YQ, Henkelman RM, Husain M, et al. Genetic deletion or pharmacological inhibition of dipeptidyl peptidase-4 improves cardiovascular outcomes after myocardial infarction in mice. Diabetes. 2010;59(4):1063-73.

21. Miyoshi T, Nakamura K, Yoshida M, Miura D, Oe H, Akagi S, et al. Effect of vildagliptin, a dipeptidyl peptidase 4 inhibitor, on cardiac hypertrophy induced by chronic beta-adrenergic stimulation in rats. Cardiovasc Diabetol. 2014;13:43.

22. Koibuchi N, Hasegawa Y, Katayama T, Toyama K, Uekawa K, Sueta D, et al. DPP-4 inhibitor linagliptin ameliorates cardiovascular injury in salt-sensitive hypertensive rats independently of blood glucose and blood pressure. Cardiovasc Diabetol. 2014;13:157.

23. Ku HC, Chen WP, Su MJ. DPP4 deficiency exerts protective effect against $\mathrm{H} 2 \mathrm{O} 2$ induced oxidative stress in isolated cardiomyocytes. PLoS One. 2013;8(1):e54518. 
24. Sakamuri S, Sperling JA, Sure VN, Dholakia MH, Peterson NR, Rutkai I, et al. Measurement of respiratory function in isolated cardiac mitochondria using Seahorse XFe24 Analyzer: applications for aging research. Geroscience. 2018;40(3):347-56.

25. Lee SY, Hsin LW, Su MJ, ChangChien CC, Ku HC. A novel isoquinoline derivative exhibits antiinflammatory properties and improves the outcomes of endotoxemia. Pharmacol Rep. 2019;71(6):1281-8.

26. Westphal D, Dewson G, Czabotar PE, Kluck RM. Molecular biology of Bax and Bak activation and action. Biochim Biophys Acta. 2011;1813(4):521-31.

27. Chaitanya GV, Steven AJ, Babu PP. PARP-1 cleavage fragments: signatures of cell-death proteases in neurodegeneration. Cell Commun Signal. 2010;8:31.

28. Soldani C, Scovassi Al. Poly(ADP-ribose) polymerase-1 cleavage during apoptosis: an update. Apoptosis. 2002;7(4):321-8.

29. Tufekci KU, Civi Bayin E, Genc S, Genc K. The Nrf2/ARE Pathway: A Promising Target to Counteract Mitochondrial Dysfunction in Parkinson's Disease. Parkinsons Dis. 2011;2011:314082.

30. Austin S, St-Pierre J. PGC1 alpha and mitochondrial metabolism--emerging concepts and relevance in ageing and neurodegenerative disorders. J Cell Sci. 2012;125(Pt 21):4963-71.

31. Fisman EZ, Tenenbaum A. Antidiabetic treatment with gliptins: focus on cardiovascular effects and outcomes. Cardiovasc Diabetol. 2015;14:129.

32. Jose T, Inzucchi SE. Cardiovascular effects of the DPP-4 inhibitors. Diab Vasc Dis Res. 2012;9(2):109-16.

33. Fadini GP, Avogaro A, Degli Esposti L, Russo P, Saragoni S, Buda S, et al. Risk of hospitalization for heart failure in patients with type 2 diabetes newly treated with DPP-4 inhibitors or other oral glucoselowering medications: a retrospective registry study on 127,555 patients from the Nationwide OsMed Health-DB Database. Eur Heart J. 2015;36(36):2454-62.

34. Scirica BM, Bhatt DL, Braunwald E, Steg PG, Davidson J, Hirshberg B, et al. Saxagliptin and cardiovascular outcomes in patients with type 2 diabetes mellitus. N Engl J Med. 2013;369(14):131726.

35. Mulvihill EE, Varin EM, Ussher JR, Campbell JE, Bang KW, Abdullah T, et al. Inhibition of Dipeptidyl Peptidase-4 Impairs Ventricular Function and Promotes Cardiac Fibrosis in High Fat-Fed Diabetic Mice. Diabetes. 2016;65(3):742-54.

36. Sell H, Bluher M, Kloting N, Schlich R, Willems M, Ruppe F, et al. Adipose dipeptidyl peptidase-4 and obesity: correlation with insulin resistance and depot-specific release from adipose tissue in vivo and in vitro. Diabetes Care. 2013;36(12):4083-90.

37. Kaifu K, Ueda S, Nakamura N, Matsui T, Yamada-Obara N, Ando R, et al. Advanced glycation end products evoke inflammatory reactions in proximal tubular cells via autocrine production of dipeptidyl peptidase-4. Microvasc Res. 2018;120:90-3.

38. Soare A, Gyorfi HA, Matei AE, Dees C, Rauber S, Wohlfahrt T, et al. Dipeptidylpeptidase 4 as a Marker of Activated Fibroblasts and a Potential Target for the Treatment of Fibrosis in Systemic Sclerosis. 
Arthritis Rheumatol. 2020;72(1):137-49.

39. Hasan AA, Hocher B. Role of soluble and membrane-bound dipeptidyl peptidase-4 in diabetic nephropathy. J Mol Endocrinol. 2017;59(1):R1-R10.

40. Zorov DB, Filburn CR, Klotz LO, Zweier JL, Sollott SJ. Reactive oxygen species (ROS)-induced ROS release: a new phenomenon accompanying induction of the mitochondrial permeability transition in cardiac myocytes. J Exp Med. 2000;192(7):1001-14.

41. Wang C, Youle RJ. The role of mitochondria in apoptosis*. Annu Rev Genet. 2009;43:95-118.

42. Li J, Yuan J. Caspases in apoptosis and beyond. Oncogene. 2008;27(48):6194-206.

43. Pintana H, Apaijai N, Chattipakorn N, Chattipakorn SC. DPP-4 inhibitors improve cognition and brain mitochondrial function of insulin-resistant rats. J Endocrinol. 2013;218(1):1-11.

44. Takada S, Masaki Y, Kinugawa S, Matsumoto J, Furihata T, Mizushima W, et al. Dipeptidyl peptidase4 inhibitor improved exercise capacity and mitochondrial biogenesis in mice with heart failure via activation of glucagon-like peptide-1 receptor signalling. Cardiovasc Res. 2016;111(4):338-47.

45. Takeda K, Sawazaki H, Takahashi H, Yeh YS, Jheng HF, Nomura W, et al. The dipeptidyl peptidase-4 (DPP-4) inhibitor teneligliptin enhances brown adipose tissue function, thereby preventing obesity in mice. FEBS Open Bio. 2018;8(11):1782-93.

46. Chae YN, Kim TH, Kim MK, Shin CY, Jung IH, Sohn YS, et al. Beneficial Effects of Evogliptin, a Novel Dipeptidyl Peptidase 4 Inhibitor, on Adiposity with Increased Ppargc1a in White Adipose Tissue in Obese Mice. PLoS One. 2015;10(12):e0144064.

47. Zillessen P, Celner J, Kretschmann A, Pfeifer A, Racke K, Mayer P. Metabolic role of dipeptidyl peptidase 4 (DPP4) in primary human (pre)adipocytes. Sci Rep. 2016;6:23074.

48. Li W, Kong AN. Molecular mechanisms of Nrf2-mediated antioxidant response. Mol Carcinog. 2009;48(2):91-104.

49. Zhou X, Wang W, Wang C, Zheng C, Xu X, Ni X, et al. DPP4 Inhibitor Attenuates Severe Acute Pancreatitis-Associated Intestinal Inflammation via Nrf2 Signaling. Oxid Med Cell Longev. 2019;2019:6181754.

50. Dinkova-Kostova AT, Abramov AY. The emerging role of Nrf2 in mitochondrial function. Free Radic Biol Med. 2015;88(Pt B):179-88.

51. Cherry AD, Suliman HB, Bartz RR, Piantadosi CA. Peroxisome proliferator-activated receptor gamma co-activator 1-alpha as a critical co-activator of the murine hepatic oxidative stress response and mitochondrial biogenesis in Staphylococcus aureus sepsis. J Biol Chem. 2014;289(1):41-52.

52. Baldelli S, Aquilano K, Ciriolo MR. Punctum on two different transcription factors regulated by PGC1alpha: nuclear factor erythroid-derived 2-like 2 and nuclear respiratory factor 2. Biochim Biophys Acta. 2013;1830(8):4137-46.

53. Navarro E, Gonzalez-Lafuente L, Perez-Liebana I, Buendia I, Lopez-Bernardo E, Sanchez-Ramos C, et al. Heme-Oxygenase I and PCG-1alpha Regulate Mitochondrial Biogenesis via Microglial Activation 
of Alpha7 Nicotinic Acetylcholine Receptors Using PNU282987. Antioxid Redox Signal. 2017;27(2):93-105.

Figures

Fig. 1

A

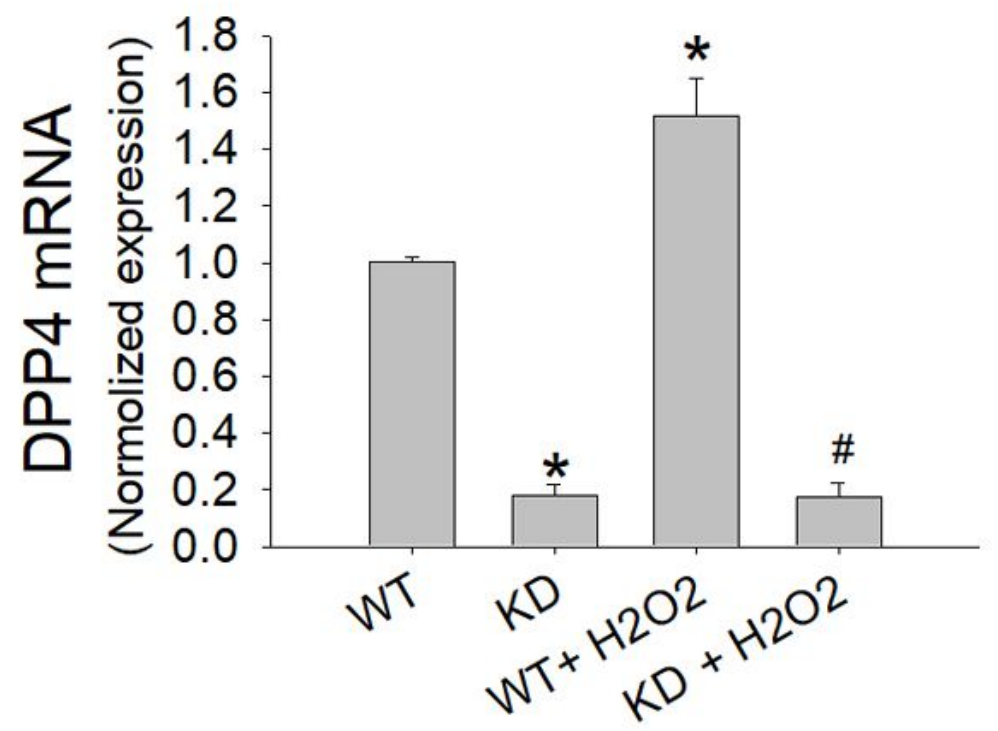

B

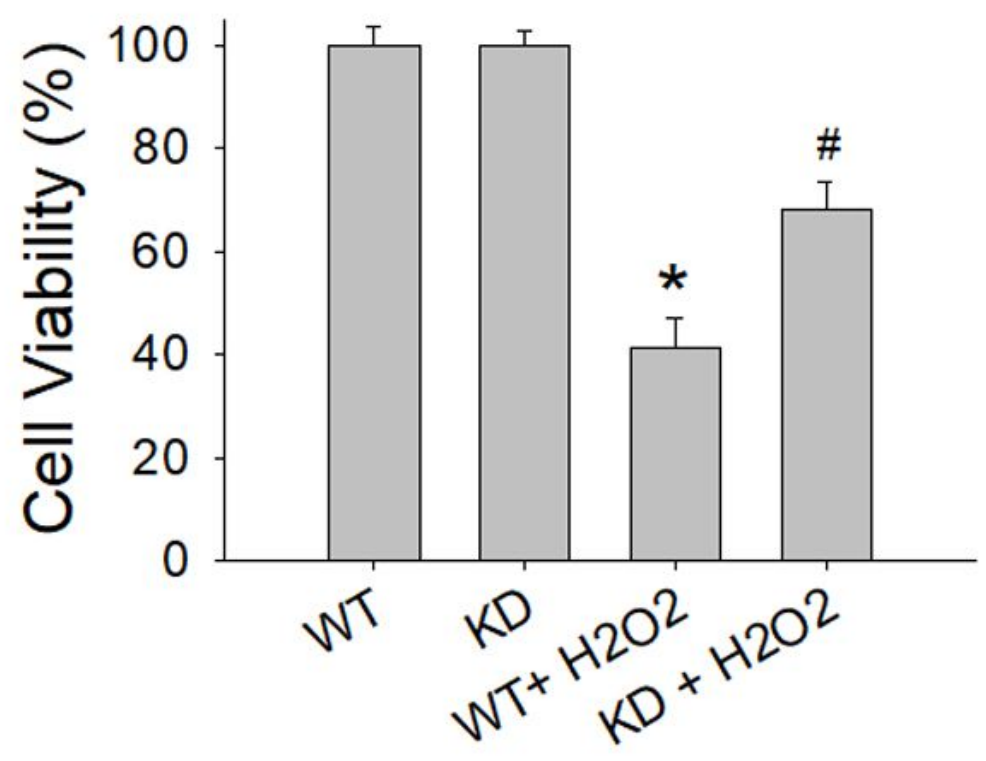

Figure 1 
Effects of DPP4 on cell viability under oxidative stress. Wild-type (WT) and DPP4 knockdown (KD) cardiomyocyte were exposed with or without $\mathrm{H} 2 \mathrm{O} 2(200 \mu \mathrm{M})$ for $24 \mathrm{~h}$. (A) DPP4 mRNA expression were measured by RT-qPCR. (B) Cell viability was tested via MTT assay. $(n=4) * p<0.05$ vs. control, $\# p<0.05$ vs. $\mathrm{WT}+\mathrm{H} 2 \mathrm{O} 2$.

Fig. 2
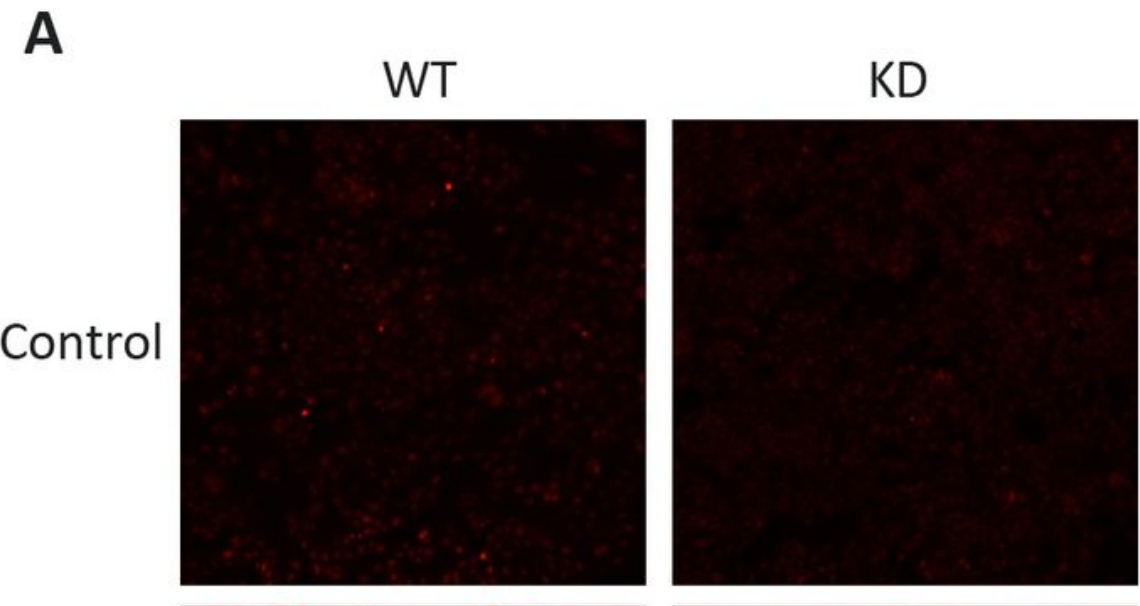

$\mathrm{H}_{2} \mathrm{O}_{2}$
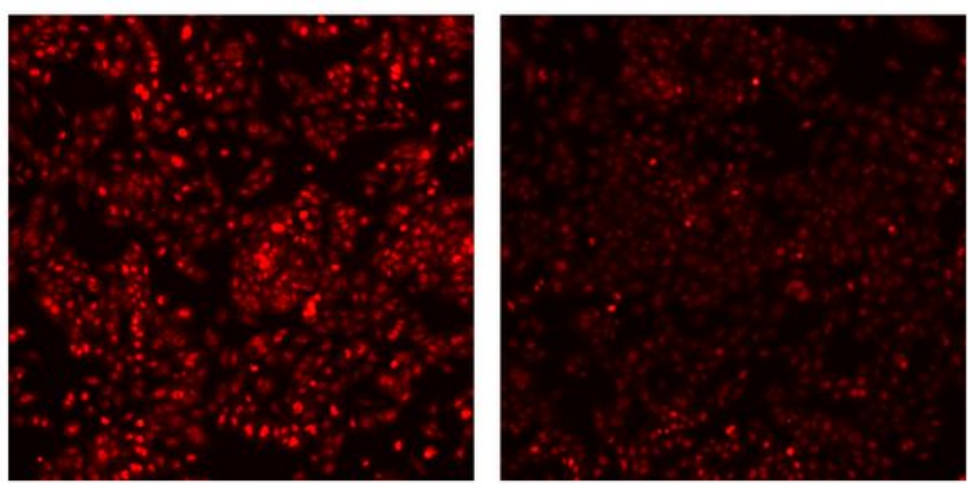

B

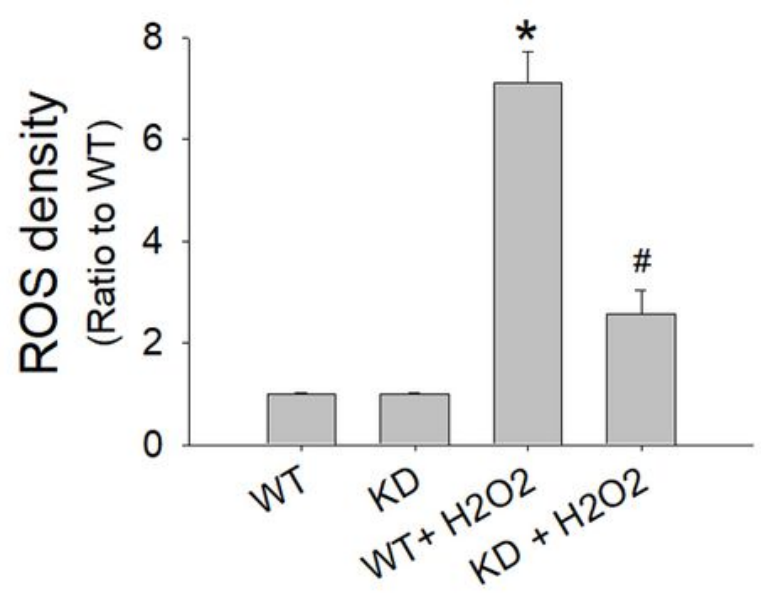

Figure 2 
Effects of DPP4 on intracellular ROS production under oxidative stress. Wild-type (WT) and DPP4 knockdown (KD) cardiomyocyte were exposed with or without H2O2 $(200 \mu \mathrm{M})$ for $24 \mathrm{~h}$. DHE staining was used for detecting intracellular ROS concentration. (A) Original microscopy photos were reported for ROS density, and (B) results of densitometry. $(n=4) * p<0.05$ vs. control, $\# p<0.05$ vs. WT+ H2O2.

Fig. 3

A

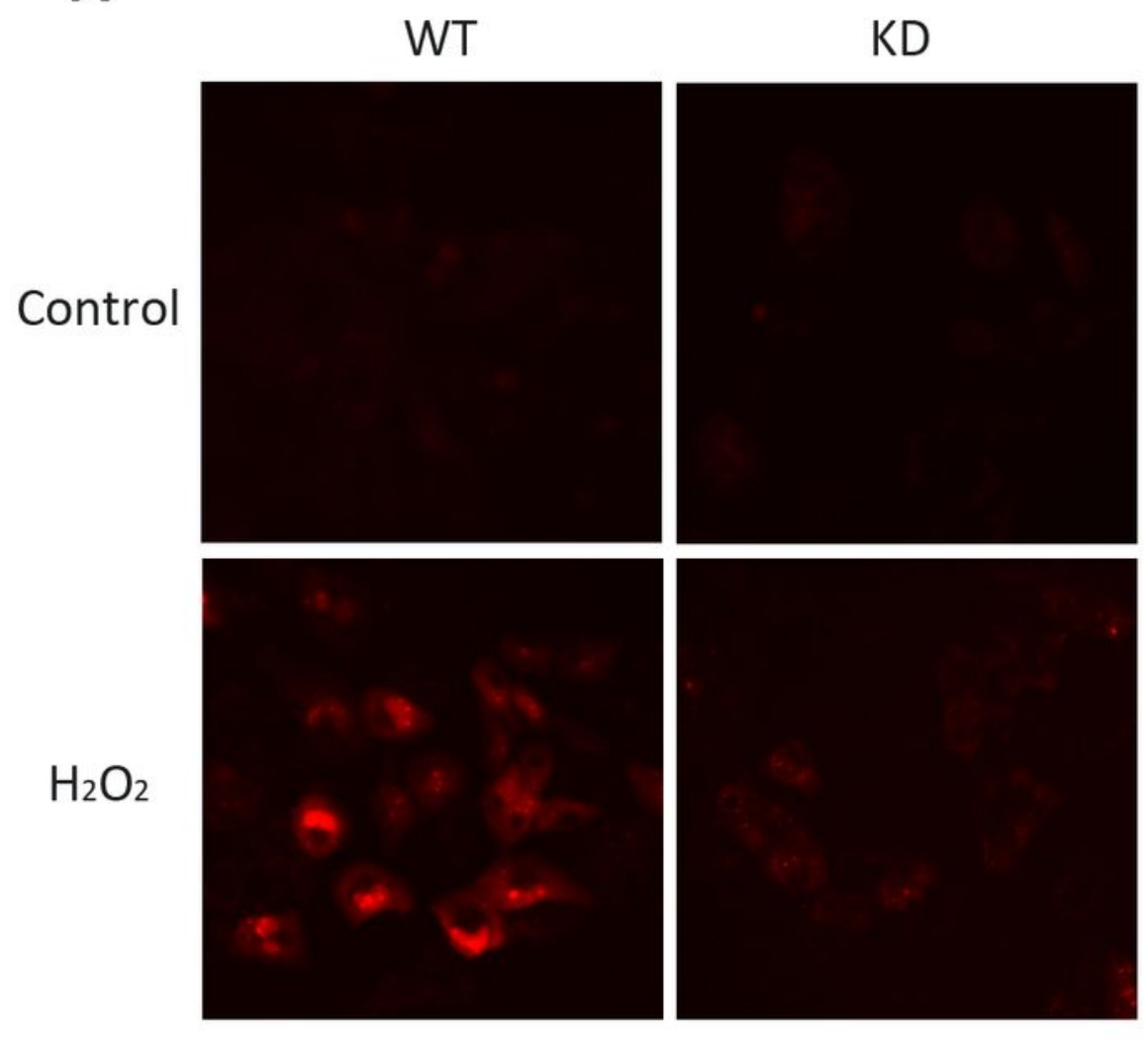

B

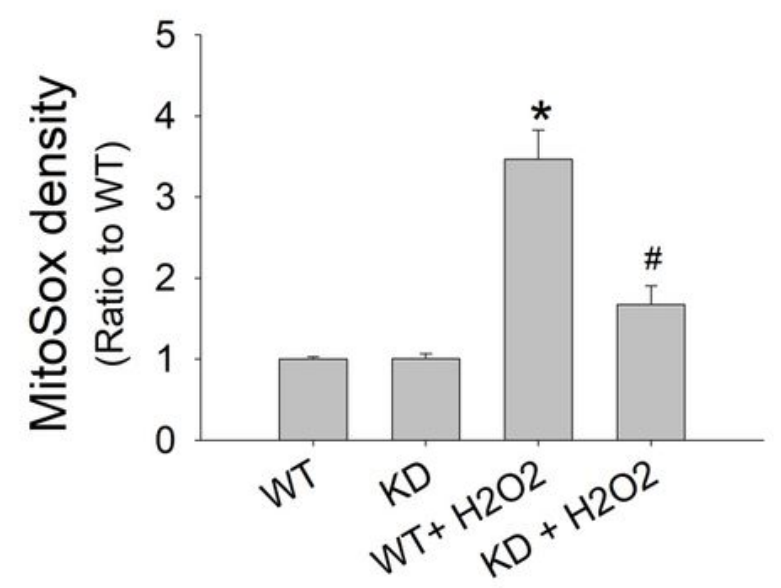

Figure 3 
Effects of DPP4 on mitochondrial superoxide production under oxidative stress. Wild-type (WT) and DPP4 knockdown (KD) cardiomyocyte were exposed with or without H2O2 (200 $\mu \mathrm{M})$ for $24 \mathrm{~h}$. MitoSOX staining was used for detecting mitochondria superoxide concentration. (A) Original microscopy photos were reported for mitochondria superoxide density, and (B) results of densitometry. $(n=4) * p<0.05$ vs. control, \# $\mathrm{p}<0.05$ vs. WT+ H2O2.

Fig. 4

A
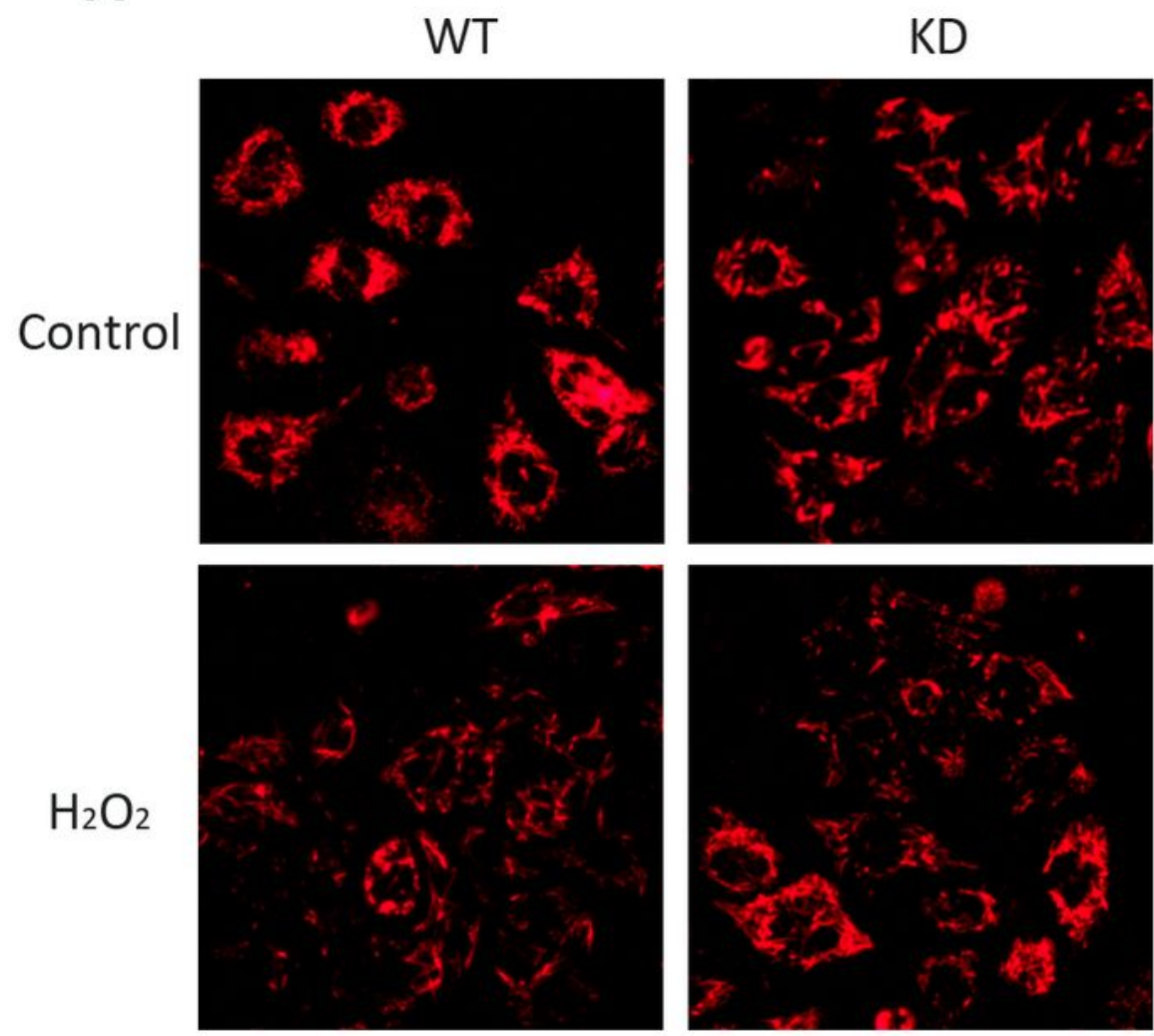

B

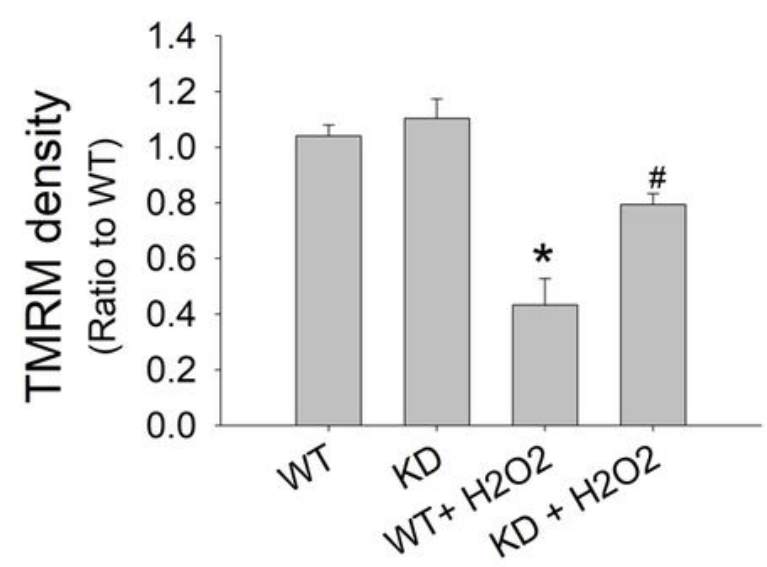

Figure 4 
Effects of DPP4 on mitochondrial membrane potential under oxidative stress. Wild-type (WT) and DPP4 knockdown (KD) cardiomyocyte were exposed with or without H2O2 $(200 \mu \mathrm{M})$ for $24 \mathrm{~h}$. TMRM staining was used for detecting mitochondrial membrane potential. (A) Original microscopy photos were reported for mitochondrial membrane potential, and (B) results of densitometry. $(n=4) * p<0.05$ vs. control, \#p< 0.05 vs. WT+ H2O2.

Fig. 5

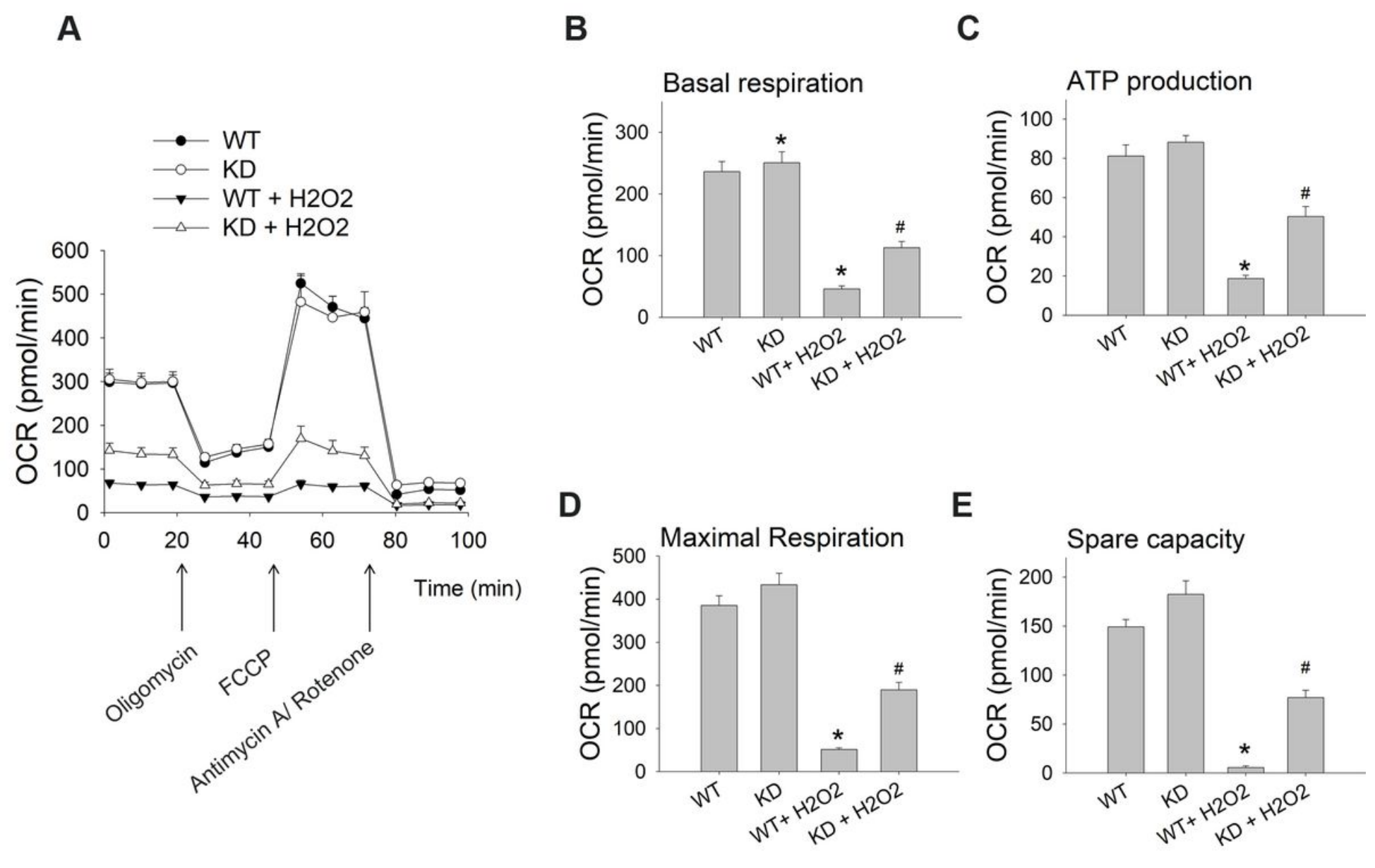

\section{Figure 5}

Effects of DPP4 on mitochondrial bioenergetics function under oxidative stress. Wild-type (WT) and DPP4 knockdown (KD) cardiomyocyte were exposed with or without $\mathrm{H} 2 \mathrm{O} 2(200 \mu \mathrm{M})$ for $24 \mathrm{~h}$. A Seahorse metabolic flux analyzer was used for measuring the rate of oxygen consumption rate (OCR). The basal OCR were first measured and subsequent injection of $1 \mu \mathrm{M}$ oligomycin, $0.5 \mu \mathrm{M} \mathrm{FCCP,} \mathrm{and} 0.5 \mu \mathrm{M}$ rotenone plus antimycin A for detecting the different parameters of OCR. (A) Mitochondrial respiration profiles of cardiomyocyte were shown. Each data point represented an OCR measurement. (B) Basal respiration, (C) ATP-linked oxygen consumption, (D) maximal oxygen consumption, (E) spare respiration capacity were calculated. $(n=5) * p<0.05$ vs. control, \# $p<0.05$ vs. WT $+\mathrm{H} 2 \mathrm{O} 2$. 
Fig. 6

A

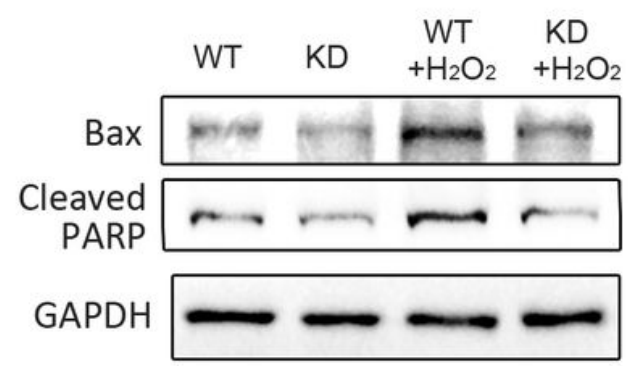

D

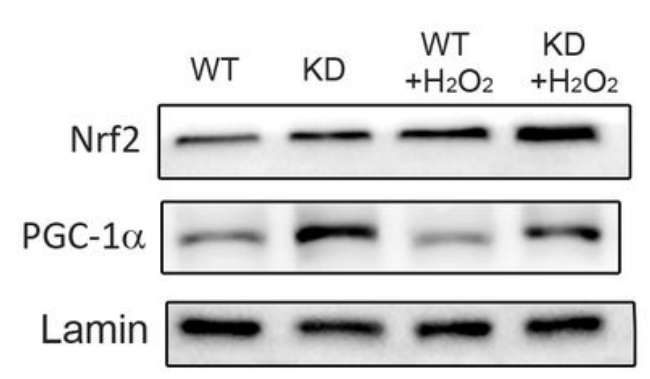

B

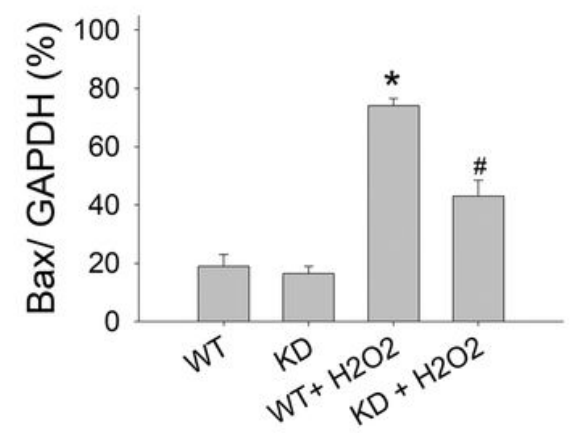

$\mathbf{E}$

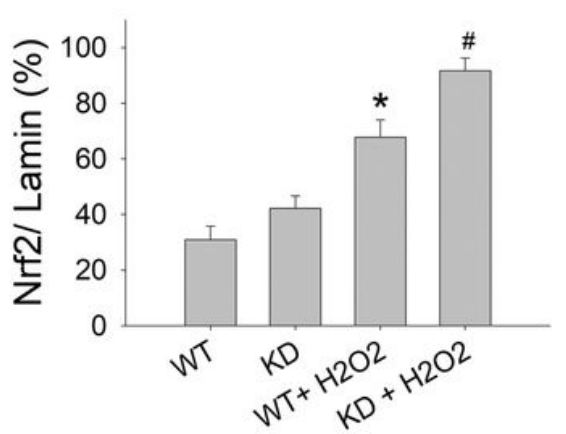

C

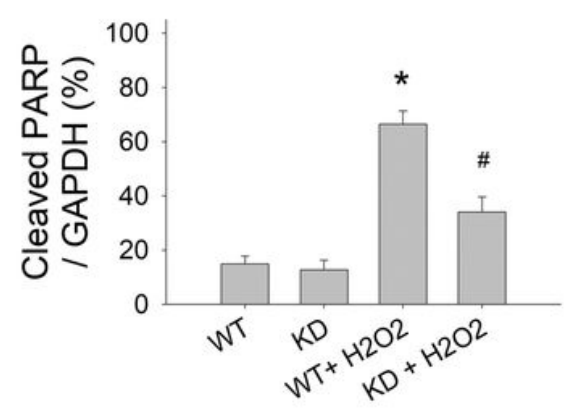

$\mathbf{F}$

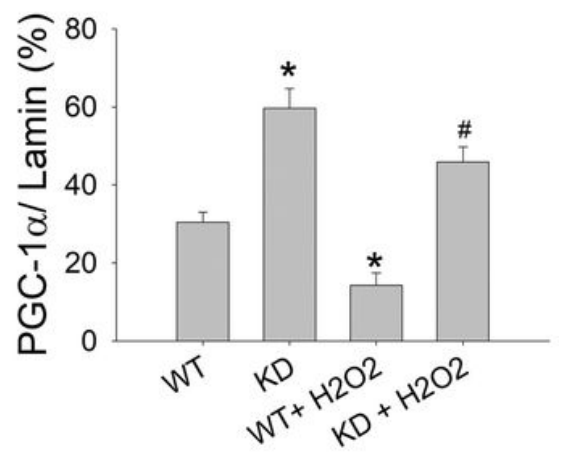

\section{Figure 6}

Effects of DPP4 on protein expression under oxidative stress. Wild-type (WT) and DPP4 knocked down (KD) cardiomyocyte were exposed with or without H2O2 $(200 \mu \mathrm{M})$ for $24 \mathrm{~h}$. Cells were harvested for total cell lysates and determined the expression of bax, cleaved PARP, and GAPDH. Cells were also harvested for the nucleus fraction of the proteins and determined the expression of Nrf2, PGC-1a, and lamin. (A) Original Western blots were shown for total cell lysates. (B) Ratios of bax to GAPDH, and (C) ratios of cleaved PARP to GAPDH were measured. (D) Original Western blots were shown for nucleus fraction of the proteins. (E) Ratios of Nrf2 to lamin, and (F) ratios of PGC-1 a to lamin were measured. $(n=4)$ * $p<$ 0.05 vs. control, \# $\mathrm{p}<0.05$ vs. WT+ $\mathrm{H} 2 \mathrm{O} 2$. 